\title{
MONITORING OF THE ELECTRON BEAM POSITION IN INDUSTRIAL LINACS
}

\author{
V.N.Boriskin*, A.N.Savchenko, V.I.Tatanov. \\ National Science Center, Kharkov Institute of Physics and Technology (NSC KIPT), Ukraine
}

\section{Abstract}

Recently the technological linear electron accelerators with the energy up to 10 and $25 \mathrm{MeV}$ and the pulse current up to $1 \mathrm{~A}$ have been developed and put into operation in the "Accelerator" R\&D Production Establishment of the National Science Center, Kharkov Institute of Physics and Technology [1]. The zone of the technological object irradiation by the accelerated electrons is created by the magnetic scanning system. Wide-aperture $(50 \times 200 \mathrm{~mm})$ magneto-induction position monitor has been designed to control the electron beam position. Signals from the monitor are used in the accelerator control system.

\section{THE OUTPUT BEAM SCANNING AND FORMING}

The technoloical object irradiation zone is formed by the magnetic scanning system [2] presented by the laminated electromagnet with spatially homogeneous field and normal boundaries on the beam input and output. The magnet is excited by the generator of the two-polar sawtooth current. The scanning system provides the required value and uniformity of the scanning as well as the measurement and operative control of the electron beam energy characteristics.

Table 1: Parameter specifications

\begin{tabular}{|l|l|}
\hline Parameter & Value \\
\hline The electron energy & $\AA=8-25 \mathrm{MeV}$ \\
\hline $\begin{array}{l}\text { The beam diameter at } \\
\text { the inlet of the scanning } \\
\text { device }\end{array}$ & $\begin{array}{l}\varnothing \approx 1 \mathrm{~cm} \\
\left(r_{0}=z_{0}= \pm 0.5 \mathrm{~cm}\right)\end{array}$ \\
\hline $\begin{array}{l}\text { The input beam angular } \\
\text { divergence }\end{array}$ & $r_{0}^{\prime}=z_{0}^{\prime} \approx 5 \cdot 10^{-3} \mathrm{radn}$ \\
\hline Energy dispersion & $\Delta E / E \approx \pm 5 \cdot 10^{-2}$ \\
\hline $\begin{array}{l}\text { The maximum angle of } \\
\text { the beam scanning }\end{array}$ & $\varphi= \pm 20^{\circ}$ \\
\hline $\begin{array}{l}\text { The effective length of } \\
\text { the magnet field }\end{array}$ & $L_{n} \cong 16.3 \mathrm{~cm}$ \\
\hline $\begin{array}{l}\text { The scanning frequency } \\
\text { (controlled) }\end{array}$ & $f=1 \ldots 3 \mathrm{~Hz}$ \\
\hline
\end{tabular}

*E-mail: boriskin@nik.kharkov.ua
The electron beam is injected to the atmosphere through a thin titanium foil. The swing of the beam track in the foil plane is about $12 \mathrm{~cm}$ in the scanning regime. The basic characteristics ofthe accelerator beam and the irradiation zone, which determine the type and construction of the scanner, are presented in the following Table 1.Uniformity of the irradiation of the longitudinal object surfaces behind an output scanner window as well as characteristics of the accelerator natural initial beam is determined mainly by the configuration and quality of the magnetic field of scanning magnet, and also by the time characteristics and the form of the sawtooth exciting current. Fig. 1 shows schematically the scanning zone from the accelerating section outlet to the output window with real boundaries of the edge field on the inlet and outlet of the scanner magnet clearance.

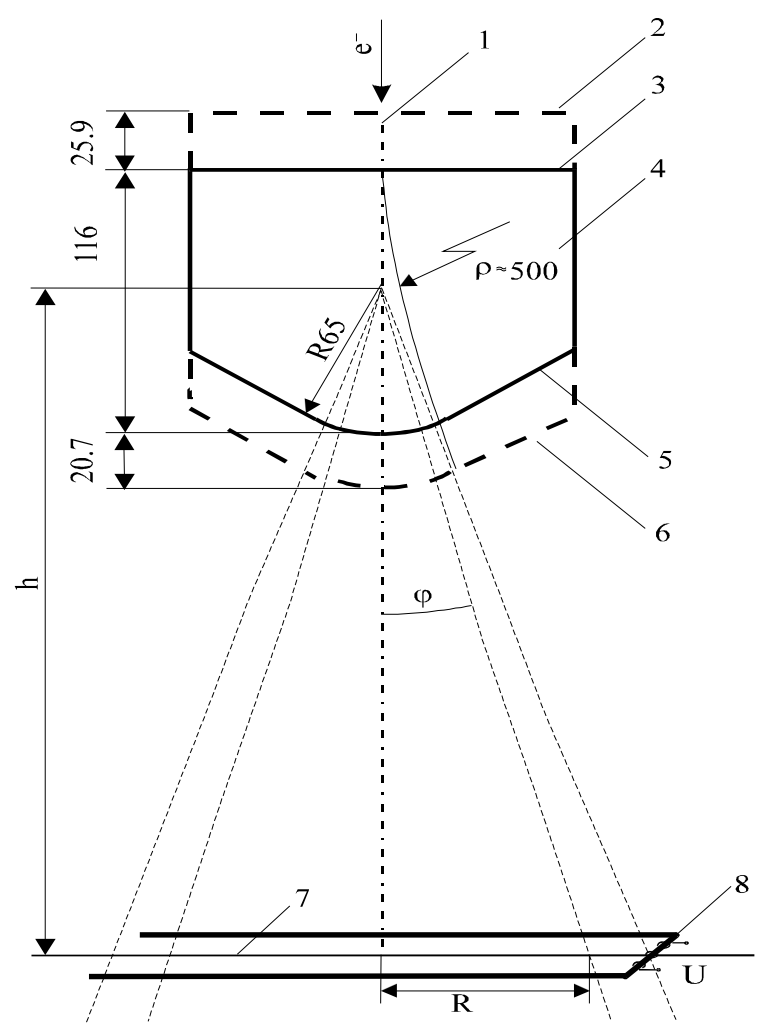

Fig. 1. The zone of beam scanning and output: 1- the accelerator axis; 2 and 6- the effective field boundaries on the inlet and outlet of the scanning magnet; 3 and 5 -the magnet boundaries (by iron); 7- the beam energy and location control plane; 8- position induction monitor. 


\section{OUTPUT BEAM POSITION MONITOR}

Induction single-coordinate monitor to determine the position of the electron beam center is placed at the accelerator output in the atmosphere at $40 \mathrm{~mm}$ from the separating foil (Fig.2). The monitor aperture is 50x200 $\mathrm{mm}$. The ferrite core cross-section is $10 \times 20 \mathrm{~mm}$. A number of winding coils is 30 . Amplitude of the signal excited in the winding by the pulse electron beam $I_{p}$ is directly proportional to $I_{p}$ and inversely proportional to the distance $\mathrm{Y}$ to the winding:

$$
Y=a U / I_{p}+b .
$$

The coefficients were determined during the bench testing when a rectangular current pulse of duration 4 mcsec was advanced along a thin long conductor (fig.2). Obtained dependence in the current working range $\left(I_{p}=0.7 \mathrm{~A}, t_{p}=4\right.$ mcsec) is presented in Fig.3.

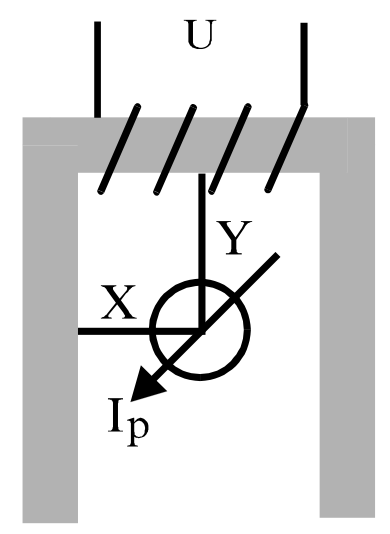

Fig. 2.The induction monitor scheme.

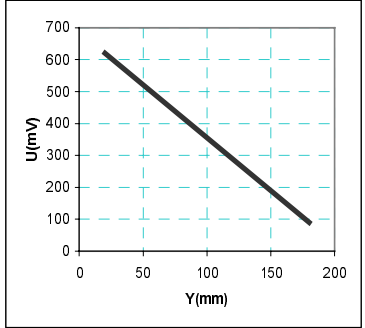

•

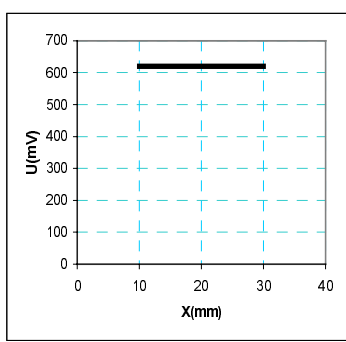

b
Fig.3. Amplitude U versus coordinate $\mathrm{Y}(\bullet)$ and coordinate $\mathrm{X}(\mathrm{b})$.

The slope of the monitor characteristic is $3,4 \mathrm{mV} / \mathrm{mm}$. After the placement of the monitor at the accelerator output the coefficients are defined more accurately by using the photometric method. At that the beam position is exposed on the glass located at the monitor aperture.

\section{AUTOMATIC CONTROL SYSTEM}

The special control system has also been developed [3] for controlling the electron beam current, energy and position as well as the control of parameters and defense of the accelerating and scanning systems from the damage caused by the beam; blocking of the modulator and klystron amplifier in the case of the intolerable operation regimes; current control in the magnetic system feeder; regulation of the phase and power of the HF signals in the injecting system; the radiation dose control and control of the target devices. The program \& technical complex consists of PC equipped with CAMAC crate or measuring channels in PC standard, synchronization unit, microprocessoroperated complexes to monitor the klystron amplifier operation, the thermostating system, and the target equipment. The measuring devices provide receiving the signal from the analog pulse probes with the 50 or 100 nsec discreteness by two or four switched channels simultaneously. The information about the accelerator system state and the electron beam parameters is displayed on the local unit terminals and on the color graphics display in the form of the triple-screen control panel (Fig.4). The operator can monitor the work of the accelerator from the PC keyboard and from the local control panels. Program units can provide the single-shot or repeated control of system parameters or give out operating commands. Simultaneously the parameters of several systems can be controlled and only one of them can be regulated. One of the system modules provides the simultaneous measurement of the values of the signals from the winding of the expanded electron beam location probe and the scanning magnet excitation current (Fig.4).

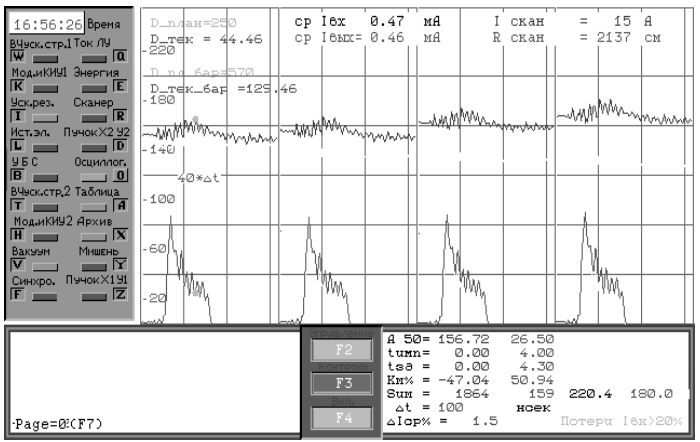

Fig.4. Videogram of the process of the electron beam energy control.

By the results of the measurements of several scanning cycles maximum $Y_{\max }$ and minimum $Y_{\min }$ values of the electron beam center deviation are calculated and the value $2 R=Y_{\max }-Y_{\min }$ is determined. 


\section{THE OPERATIVE CONTROL OF THE ELECTRON ENERGY}

It was shown [4] that the electromagnet of the output device can be used for the operative control of the electron energy. The proposed technique is based on the relationship between the total energy of the charged particle $E_{n}$ and the magnetic field $H \rho$ :

$$
H \rho=\frac{1}{e c} \sqrt{E_{n}^{2}-E_{0}^{2}},
$$

where $c$ - is the light speed, $e$ and $E_{0} 0.5 \mathrm{MeV}$ - are the electron charge and rest energy, respectively, $\rho$ - is the radius of the particle trajectory in the magnetic field with intensity $H$. The following expression for the kinetic energy of the electron can be obtained by a simple transformation of (1) taking into account dimensions and units of measurement:

$$
E_{k}=\sqrt{E_{0}^{2}+\left(3 \cdot 10^{-4} H \rho\right)^{2}}-E_{0}
$$

where $E_{k}$ is counted in $\mathrm{MeV}, \rho$ in $\mathrm{cm}$. One can see that to measure the $E_{k}$ value it is enough to determine two values: the intensity of the deflecting magnetic field $H$ and the radius of the electron turn in this field $\rho$. However, while working with an accelerated beam the direct measurement of the specified characteristics, especially $\rho$, is very difficult. That is why the technique is based on the measurement of the linear and angular parameters - the effective length of the deflecting magnetic field $L_{n}$ (taking into account the scattered fields on the inlet and outlet of the magnet), and the geometry of the scanning zone (Fig. 1). These parameters are measured in advance and do not change their values during the experiment. The excitation current force of the scanning electromagnet is obtained from the curve of magnetization $H=f(I)$. Noticing that for the small angles of the beam deflection $\left(\varphi \leq 20^{\circ}\right)$ the following relationship is valid

$$
\rho=\frac{L_{n}}{\sin \varphi},
$$

we arrive at the practical formula for $E_{k}$ :

$$
E_{k}=\sqrt{E_{0}^{2}+\frac{k^{2} I^{2}}{\sin ^{2} \varphi}}-E_{0} \text {, }
$$

where $k$ is the constant accounting the relationship between $H, I$ and $L_{n}$ for a specific magnet and is determined by bench testing. From Fig. 1 one can see that if the value of the electron beam deflection from the axis $(R)$ on the plane located at distance $h$ from the magnet is found by using any appropriate method and the value $I$ is known, the most expectable value of $E_{k}$ can be obtained. In this case

$$
\varphi=\operatorname{arctg}(R / h)
$$

The monitor described above enables to evaluate the current value $R$ with an error of about 3\%. The amplitude of the electromagnet excitation current $I$ is determined at the same time, and then the value $E_{k}$ is calculated. The videogram of the process of the electron beam energy control is shown in Fig.5.

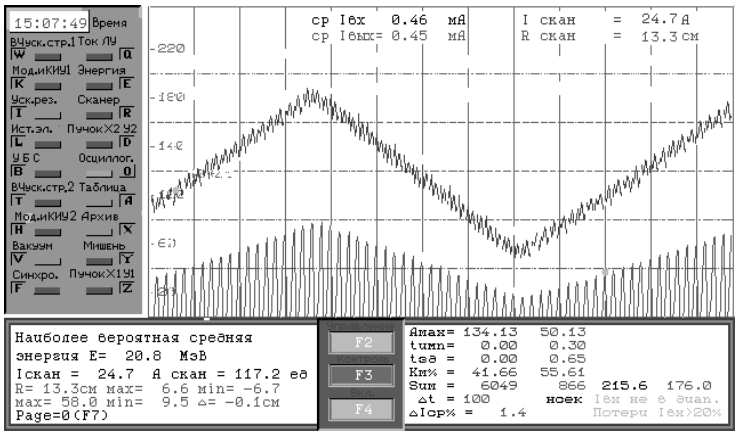

Fig.5. Videogram of the process of the electron beam energy control.

The evaluation of value $R$ and the electron energy is performed without a denormalization of the accelerator working regime and takes about $2 \mathrm{sec}$.

\section{REFERENCES}

[1] A.N.Dovbnya et al. Electron Linacs Based Radiation Facilities of Ukrainian National Science Center "KIPT / Bulletin of the American Physical Society, May 1997, V.42, No.3, p.1391."

[2] A.N.Dovbnya, et al. , "The Output Beam Scanning and Forming in the Multipurpose Electron Accelerators of KIPT", VANT,Series: Nucleic Physics, 1997, vol 1(28). p. 114-121.

[3] V.N.Boriskin et al. Control system for a linear resonance accelerator of intense electron beams / Nucl. Instr. and Meth. in Phys. Res A 352 (1994) 61-62.

[4] V.N.Boriskin, A.E.Tolstoy, V.L.Uvarov et al., "Automatic Control of the Electron Energy in the Technological Linear Electron Accelerators", Digest of the XIV Meeting on the Accelerated Particles, Protvino,Russia, 1994, vol.2, pp.97-98 